\title{
Sugar intake in Scottish children (Full4Health Study)
}

\author{
E. O'Leary ${ }^{1}$, W. Buosi ${ }^{2}$, D. Crabtree ${ }^{2}$, G. Horgan ${ }^{3}$, C. Fyfe ${ }^{2}$ and A.M. Johnstone ${ }^{2}$ \\ ${ }^{1}$ University College Dublin, Belfield, Dublin 4, Ireland, \\ ${ }^{2}$ The Rowett Institute, University of Aberdeen, AB25 2ZD, UK and \\ ${ }^{3}$ Biomathematics and Statistics Scotland, Aberdeen, AB25 2ZD, UK.
}

More than $32 \%$ of children in Scotland aged 7-11yrs, are overweight or obese ${ }^{(1)}$. Elevated dietary sugar intake, especially "free sugars", has been linked to increased risk for obesity in children ${ }^{(1)}$. The recently published Scientific Advisory Committee for Nutrition $(\mathrm{SACN})$ report $^{(2)}$ sets out new dietary recommendations for children. It advises on carbohydrate $(\mathrm{CHO} \%)$, total sugar (g), free sugar (for which non-milk extrinsic sugar (NMES) \%, acts as a proxy for free sugar ${ }^{(3)}$ ) and dietary fibre (g) intake, evidenced by health implications. One focus is the prevention of overweight/obesity. The aim of the present study was to investigate if current and habitual intakes in Scottish children approach the current recommendations. We analysed diet recall data, collected over 2015/ 2016 (Full4Health, www.full4health.eu), using a 24hour recall method. We analysed children's (8-10yrs) habitual energy, CHO, sugar (total sugar and free sugar) and dietary fibre. This is the same methodology applied in the National Diet and Nutrition Survey for Scotland $(2008-12)^{(4)}$ which reports previously that NMES was $14.7 \%$ in $2011 / 2012^{(4)}$. This is some way from the $<5 \%$ new free sugar intake levels proposed ${ }^{(2)}$.

This study was approved by the North of Scotland Research Ethics committee. Participants and their parents gave informed consent. Participants $\left(\mathrm{n}=38,16\right.$ girls average BMI $18.0 \mathrm{~kg} / \mathrm{m}^{2}$ and age $8.7 \mathrm{yrs}, 22$ boys average BMI $16.8 \mathrm{~kg} / \mathrm{m}^{2}$ and age $\left.8.7 \mathrm{yrs}\right)$ completed four 24 hour diet recalls with estimated food portion size. Energy and nutrient intakes were calculated from food composition tables (NetWisp software, V3.0) and averaged over the four non-consecutive recording days. The AOAC method was applied for dietary fibre assessment ${ }^{(2)}$.

Statistical analysis confirmed that, on average, boys consumed more energy, dietary fibre and carbohydrates than girls, however girls consumed more total sugar and NMES than boys.

On average, the children consumed $13.5 \%$ energy as NMES. This was particularly noticeable in the girls, who consumed $3 \cdot 1 \%$ more NMES than boys $(p=0.02)$. Adherence to the new recommendations in this small cohort $(<5 \%)$ was non-existent, only six participants had a NMES $<10 \%$, all being boys (range 6.0-22.9\%). On average, participants exceeded the $50 \%$ recommendation of total energy coming from carbohydrates. On average, dietary fibre intake, $12.6 \mathrm{~g} / \mathrm{d}$ and $14.4 \mathrm{~g} / \mathrm{d}$ for the girls and boys respectively, did not meet the current SACN guidelines of $20 \mathrm{~g} / \mathrm{d}$.

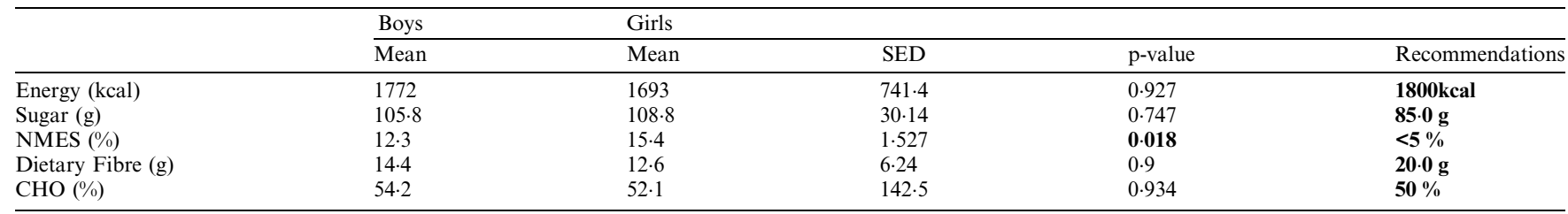

Our results reveal the average Scottish child is failing to meet the UK guidelines for dietary fibre. However our data suggests that there is an improvement from the NDNS report ${ }^{(4)}$ in 2012 where 4-10 yr olds mean intake of dietary fibre was only $10 \cdot 5 \mathrm{~g}$. Our data suggests that there is a decline in NMES intake for children in the Grampian region. Mean intakes of NMES decreased from $16.9 \%$ of total energy in $2008^{(5)}$ to $14.8 \%$ in $2012^{(4)}$ and our results now show the average child intake of NMES to be further reduced to $13.5 \%$.

Our data indicates that Scottish children are failing to meet recommendations in relation to dietary fibre, total sugar and non-milk extrinsic sugars. However, girls in particular are substantially exceeding the recommended intakes of total sugar and non-milk extrinsic sugars. Gender specific interventions may be useful for future research and evaluation to reduce sugar intake in girls and boys.

The work is funded by the European Union Seventh Framework Programme (FP7/2007-2013) under grant agreement $n^{\circ} 266408$ for the "Full4Health" grant.

1. Public Health England. (2014) Health Risks of Childhood Obesity. PHE, London.

2. The Scientific Advisory Committee on Nutrition (2015) Carbohydrates and Health.

3. Gibson S, Francis L et al. (2016) British Journal of Nutrition 116(7).

4. National Diet and Nutrition Survey: Results from Years 1-4 (combined) of the Rolling Programme 2008/09-2011/12 Report.

5. Sheehy C, McNeill G, et al. (2008) Survey of Sugar Intake among Children in Scotland, Food Standards Agency. 